\title{
ANÁLISE DE SENSIBILIDADE DE VARIÁVEIS E PARÂMETROS DE EQUAÇÕES DE FATOR DE SEGURANÇA
}

\author{
Felipe Costa Abreu Lopes (a) \\ (a) IFSP - Campus Jundiaí e doutorando em Geografia - UFPR, fcalopes@ifsp.edu.br
}

Eixo: Geografia física e desastres naturais

\begin{abstract}
Resumo
Equações matemáticas para estipular áreas propícias aos movimentos de massa, especialmente escorregamentos translacionais, são ferramentas frequentes na análise de estabilidade de encostas. Existem muitas equações criadas em diversas partes do mundo para este fim que são empregadas no Brasil, tanto na forma de modelos fechados como na de equações livres aonde o usuário tem mais controle sobre as variáveis. Cada equação traz as características do pesquisador e do local de onde foi elaborada, deste modo nem todas podem ser consideradas aptas para uso em um ambiente de clima tropical (em sua maioria) como o Brasil, onde determinadas variáveis podem requerer um peso maior ou menor do que o que está imposto em determinado modelo. Desta maneira esse trabalho faz uma análise de sensibilidade dos fatores de duas equações abertas de Fator de Segurança (FS) para evidenciar essas diferenças regionais permeadas nas equações e despertar uma discussão sobre o assunto.
\end{abstract}

Palavras chave: Fator de segurança, análise de sensibilidade, estabilidade de encosta

\section{Introdução}

As análises de sensibilidade são estimativas baseadas em variáveis e parâmetros de uma equação que têm o objetivo de verificar o efeito da variação de seus valores, dentro de um intervalo prédeterminado, sobre o resultado final dessa equação. Neste trabalho entende-se por variáveis e parâmetros de uma equação as suas definições de acordo com a matemática (WEBER, 1986) onde parâmetros são constantes paramétricas, que têm o valor constante para determinado problema e seus valores não mudam no tempo ou têm mudanças insignificantes. Variáveis são valores que mostram o estado momentâneo de um sistema e podem assumir qualquer valor dentro de um intervalo determinado com variação significativa ao longo do tempo. Em uma equação de Fator de Segurança (FS), por exemplo, declividade e espessura do solo são parâmetros, enquanto nível freático e precipitação são variáveis.

As equações de fator de segurança trabalham com uma série de variáveis e parâmetros complexos e interdependentes, conhecer qual desses valores envolvidos têm mais ou menos peso no resultado final pode auxiliar o pesquisador em uma série de decisões como, por exemplo, a escolher por detalhamentos maiores de uma variável em detrimento de outra na sua pesquisa, escolha de locais para maior quantidade de coleta de material em campo ou até a troca da equação que está sendo usada por outra que dê mais peso a uma variável ou parâmetro de seu interesse. 
A aplicação da análise de sensibilidade é muito difundida em estudos acadêmicos e trabalhos técnicos, principalmente em áreas de economia e negócios, aplicada para verificar as influências sobre investimentos ou em estudos sobre finanças. Sua aplicação na informática também é facilmente encontrada na literatura com o uso de modelos matemáticos para medir a eficiência de softwares. Outras áreas que se utilizam desse tipo de análise são as engenharias, as áreas sociais e estudos ambientas

A área ambiental, apesar de contar com menor números de trabalhos, também faz uso da análise de sensibilidade, principalmente na aplicação de modelos matemáticos, a exemplo do trabalho realizado por Agam et al (2016) sobre a sensibilidade de variáveis geotécnicas na estabilidade de uma encosta usando o método de análise de sensibilidade de Monte Carlo embutido no software Slice 6.0. Entre os modelos usados para cálculo de estabilidade de encosta, o modelo probabilístico LISA (HAMOND et al, 1992) também possui um método em seu programa para análise de sensibilidade dos fatores que afetam a ocorrência de movimentos de massa. Outro exemplo do uso da análise de sensibilidade em estudos ambientais é na calibração de modelos, por exemplo o modelo chuva-vazão TOPMODEL (BEVEN e KIRKBY, 1978) que entre suas funcionalidades faz uso do método de Monte Carlo para realizar uma calibração automática dos dados de entrada.

A aplicação da análise de sensibilidade não implica em um cálculo de risco, portanto esta não vai identificar as áreas propicias aos movimentos de massa em um estudo de estabilidade de encosta, mas vai evidenciar quais fatores têm mais relevância e devem ser mais levados em consideração quando houver alguma alteração ambiental, auxiliando nas suas identificações, na escolha e no desenvolvimento de formas de mitigação mais adequadas aos parâmetros e variáveis envolvidos.

\section{Metodologia}

Visando a identificação das variáveis e parâmetros com mais influência sobre equações de fator de segurança, uma análise de sensibilidade foi realizada sobre duas equações selecionadas (equação 1 e 2). A primeira foi descrita por Fiori e Carmignani (2009) e foi escolhida por ser uma equação que trabalha com solos e umidade, permitindo a entrada de espessuras de perfil seco e úmido de solo, além da coesão do solo, ângulo de atrito interno, pesos específicos do solo e inclinação da encosta. A segunda é uma equação desenvolvida por Sidle (1987) para cálculo de estabilidade de encosta em bacias de zero ordem e foi escolhida por apresentar as mesmas variáveis e parâmetros da equação 1 e, dessa maneira, permitir uma comparação entre as duas e focalizar sobre a análise de sensibilidade. 


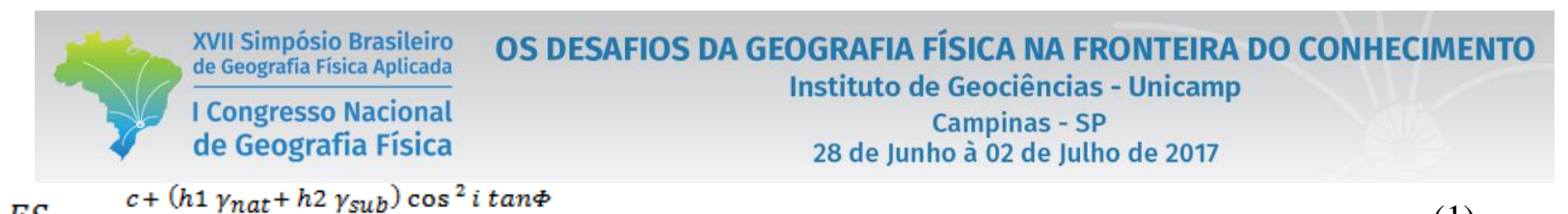

$F S=\frac{c+\left(h 1 \gamma_{n a t}+h 2 \gamma_{s u b}\right) \cos ^{2} i \tan \Phi}{\left(h 1 \gamma_{n a t}+h 2 \gamma_{s u b}+h 2 \gamma_{a}\right) \operatorname{sen} i \cos i}$

$F S=\frac{c+\Delta c\left\{\left[h 1 \gamma_{\text {nat }}+h 2 \gamma_{\text {sat }}\right] * Z \cos ^{2} i+W_{t} \cos i\right\} \tan \Phi}{\left[h 1 \gamma_{\text {sub }}+h 2 \gamma_{\text {sat }}\right] * Z \operatorname{sen} i \cos i+W_{t} \operatorname{sen} i}$

Onde: $c$ representa a coesão do solo $(\mathrm{kPa}), \Delta c$ é o valor da coesão das raízes $(\mathrm{kPa}), \gamma_{s u b}$ é o peso específico do solo submerso $\left(\mathrm{kN} / \mathrm{m}^{3}\right), \gamma_{s a t}$ é o peso específico do solo saturado $\left(\mathrm{kN} / \mathrm{m}^{3}\right), \gamma_{a}$ é o peso específico da água $\left(\mathrm{kN} / \mathrm{m}^{3}\right), W_{t}$ é o peso da vegetação, $\Phi$ é o ângulo de atrito interno do solo (graus), $i$ é a declividade da encosta (graus), $h l$ é a espessura do solo seco, $h 2$ é a espessura do solo saturado e $Z$ é a espessura total do solo.

Os fatores das equações apresentados acima foram organizados de acordo com sua classificação (variável ou parâmetro) como exposto na tabela 1. Os valores mínimos e máximos apresentados para cada um têm origem em medições realizadas em laboratório com amostras colhidas em campo $\left(\gamma_{s u b}, \gamma_{s a t}\right.$, $\gamma_{a}, \Phi, c$ e Z) e geoprocessamento e cálculo $(h 1, h 2$ e $i$ ) de acordo com Lopes (2013). O valor de $i$ foi delimitado entre um mínimo de $24^{\circ}$ (valor mais baixo do ângulo de atrito interno) e $80^{\circ}$ (valor máximo encontrado na bacia do Gigante). Percebeu-se em testes durante o desenvolvimento do trabalho que o uso de declividades muito baixas levava a um valor de FS muito elevado, distorcendo a análise de sensibilidade. Além disso valores muito baixos não representam as áreas de encosta onde ocorrem os movimentos de massa.

Para essa análise de sensibilidade os parâmetros $\Delta c$ (coesão de raízes) e $W_{t}$ (peso da vegetação) não foram levados em consideração. O parâmetro de coesão de raízes foi excluído da análise porque o local onde ocorre a ruptura geralmente está situado abaixo da linha de raízes. O peso da vegetação é um parâmetro calculado de maneira empírica e segundo próprio autor da equação 2 (SIDLE, 1987) é o fator com menos sensibilidade entre todos os analisados na estabilidade da encosta, por isso não fez parte da análise de sensibilidade. 
Tabela 1 - Variáveis e parâmetros usados na análise de sensibilidade. Fonte: autor.

\begin{tabular}{l|l|l|l}
\hline Parâmetros & Valor mínimo & Valor médio & Valor máximo \\
\hline Declividade $\left(^{\circ}\right)$ & 24 & 52 & 80 \\
\hline Espessura do solo $(\mathrm{m})$ & 0 & 2,50 & 5,0 \\
\hline Ângulo de atrito $\left(^{\circ}\right)$ & 24,50 & 29,35 & 34,10 \\
\hline Peso específico natural $\left(\mathrm{kN} / \mathrm{m}^{3}\right)$ & 13,40 & 14,95 & 16,50 \\
\hline Peso específico submerso $\left(\mathrm{kN} / \mathrm{m}^{3}\right)$ & 20,20 & 21,65 & 23,10 \\
\hline Peso específico saturado $\left(\mathrm{kN} / \mathrm{m}^{3}\right)$ & 21,20 & 22,65 & 24,10 \\
\hline Variáveis & Valor mínimo & Valor médio & Valor máximo \\
\hline Nível freático $(\mathrm{m})$ & 0 & 2,50 & 5,0 \\
\hline Coesão $(\mathrm{kPa})$ & 0 & 7,62 & 15,25 \\
\hline
\end{tabular}

O nível freático e a coesão foram considerados variáveis, pois seus valores mudam rapidamente em um evento de precipitação intensa, o principal gatilho causador de movimentos de massa no Brasil.

$\mathrm{Na}$ análise de sensibilidade cada variável e parâmetro tem seus valores variando uniformemente do valor mínimo ao máximo por incremento, enquanto os outros valores ficam inalterados em seu valor médio (obtido pela média aritmética dos valores mínimos e máximos de cada parâmetro e variável). As variáveis e parâmetros citados acima tiveram os intervalos de valores divididos em 50 intervalos iguais e o incremento adicionado a cada simulação de sensibilidade tem o valor de 1/50 do intervalo de valores para cada parâmetro/variável.

Foram feitas 50 simulações para cada parâmetro/variável totalizando 400 simulações, cada uma resultando em um valor de fator de segurança próprio. Os valores resultantes foram organizados em gráficos para a posterior análise e comparação entre as equações.

\section{Resultado}

Os resultados das análises de sensibilidade das variáveis e parâmetros das equações de FS podem ser vistos nos gráficos a seguir (Figura 1 e 2). Os dados apresentados foram discretizados por cor e apresentados com seus valores em porcentagem (eixo das abscissas) para facilitar sua comparação, já que 


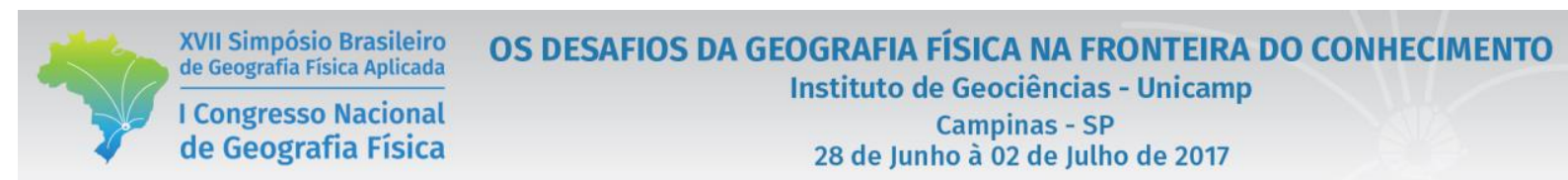

seus valores e intervalos de análise diferem entra cada um. $\mathrm{O}$ valor de $50 \%$ representa o valor médio de cada variável/parâmetro. O eixo das ordenadas apresenta os valores do fator de segurança com o valor um (1) destacado por uma linha pontilhada representando o limite de equilíbrio de estabilidade (acima da unidade, estável e abaixo instável).

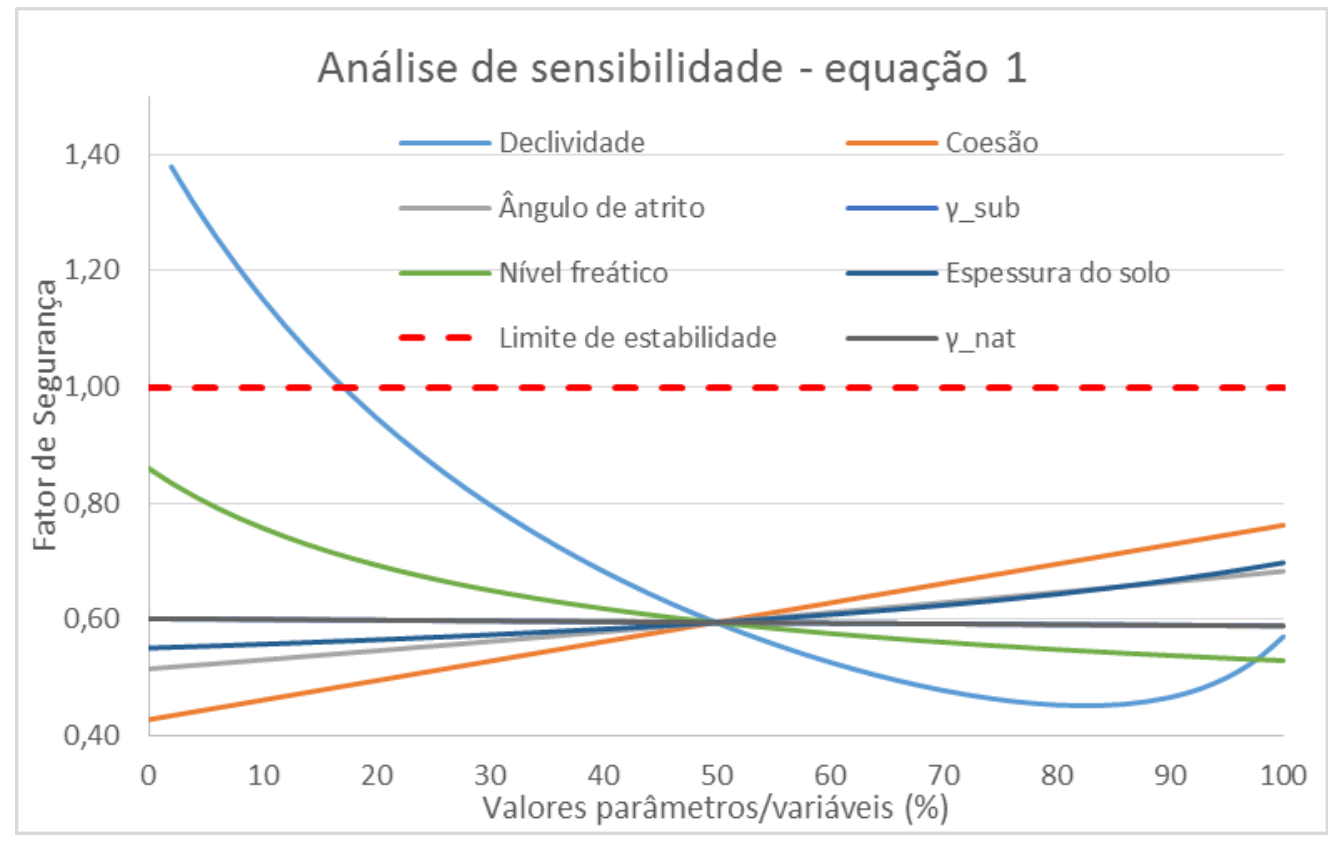

Figura 1 - Análise de sensibilidade da equação 1. Fonte: autor.

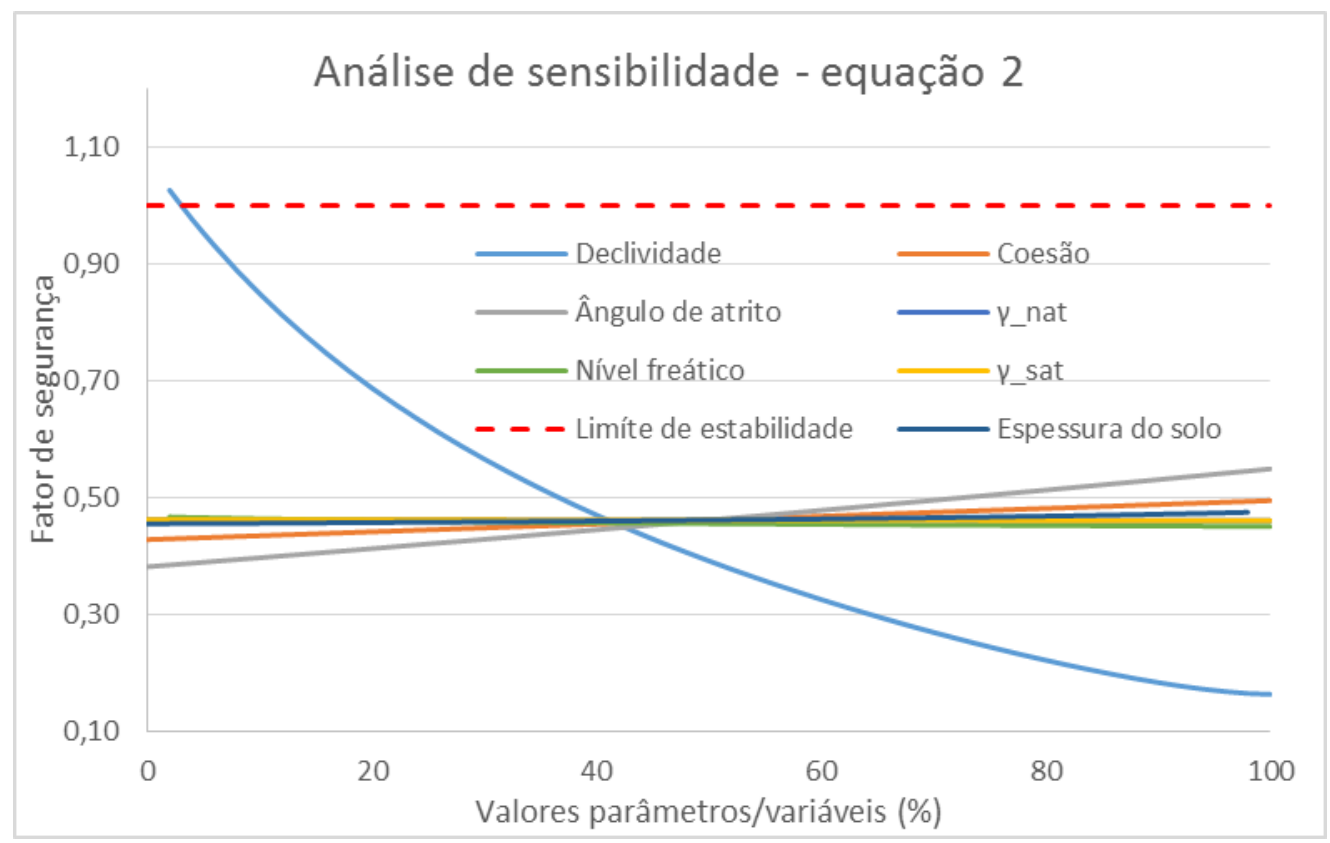

Figura 2 - Análise de sensibilidade da equação 2. Fonte: autor. 
A partir da análise do gráfico da figura 1, percebe-se um grupo de variáveis/parâmetros que têm seus valores diretamente proporcionais aos valores de FS: coesão, ângulo de atrito interno e espessura do solo. Essas variáveis/parâmetros, portanto, contribuem mais para a estabilidade da encosta quanto maior forem seus valores.

Outro grupo apresenta valores inversamente proporcionais ao FS: nível freático, peso específico natural do solo e peso específico submerso do solo. Essas variáveis/parâmetros, portanto, contribuem menos para a estabilidade da encosta quanto maior forem seus valores.

A declividade apresentou um comportamento fora do esperado apresentando um aumento do valor de FS para seus maiores valores a partir de um certo ponto, enquanto que, teoricamente, seria esperado que uma declividade mais acentuada resultaria sempre em uma instabilidade maior, porém como a análise de sensibilidade é feita com uma variável/parâmetro por vez (enquanto todas os outros permanecem travados em seu valor médio), a partir de um certo ponto observa-se essa inflexão na curva que representa a declividade em decorrência da configuração da própria equação do FS.

O gráfico da figura 2 apresentou como grupo de variáveis/parâmetros com valores diretamente proporcionais ao valor de FS a coesão, o ângulo de atrito interno e a espessura do solo. Portanto o aumento de seus valores aumenta, também, o valor do FS.

O grupo de variáveis/parâmetros cujo valores diminuem e consequentemente aumenta o valor de FS é representado pela declividade, peso específico natural do solo, peso específico saturado do solo e nível freático. Assim como no gráfico da equação 1, a declividade apresentou comportamento anômalo a partir de certo ponto pelos mesmo motivos explicados acima relativos à configuração da equação e da análise de sensibilidade.

Entre as variáveis analisadas, as suas sensibilidades foram calculadas por meio da diferença entre seus valores mínimos e máximos de FS obtidos. Dentro deste limite a maior sensibilidade foi verificada no parâmetro declividade seguida pelas variáveis nível freático e coesão para o gráfico da equação 1. Já no gráfico da equação 2 as maiores sensibilidades foram notadas no parâmetro declividade, seguido por ângulo de atrito e coesão. A sensibilidade de cada variável é apresentada nas tabelas 2 e 3. 
Tabela 2 - Sensibilidade de cada parâmetro/variável para a equação 1. Fonte: autor.

\begin{tabular}{l|c}
\hline Variável & Variação do FS \\
\hline Declividade $\left(^{\circ}\right)$ & 0,81 \\
\hline Nível freático $(\mathrm{m})$ & 0,33 \\
\hline Coesão $(\mathrm{kPa})$ & 0,33 \\
\hline Ângulo de atrito $\left(^{\circ}\right)$ & 0,17 \\
\hline Espessura do solo $(\mathrm{m})$ & 0,15 \\
\hline Peso específico natural $\left(\mathrm{kN} / \mathrm{m}^{3}\right)$ & 0,01 \\
\hline Peso específico submerso $\left(\mathrm{kN} / \mathrm{m}^{3}\right)$ & 0,01 \\
\hline
\end{tabular}

Tabela 3 - Sensibilidade de cada parâmetro/variável para a equação 2. Fonte: autor.

\begin{tabular}{l|c}
\hline Variável & Variação do FS \\
\hline Declividade $\left(^{\circ}\right)$ & 0,86 \\
\hline Ângulo de atrito $\left(^{\circ}\right)$ & 0,17 \\
\hline Coesão $(\mathrm{kPa})$ & 0,07 \\
\hline Espessura do solo $(\mathrm{m})$ & 0,02 \\
\hline Nível freático $(\mathrm{m})$ & 0,01 \\
\hline Peso específico natural $\left(\mathrm{kN} / \mathrm{m}^{3}\right)$ & 0,002 \\
\hline Peso específico saturado $\left(\mathrm{kN} / \mathrm{m}^{3}\right)$ & 0,002 \\
\hline
\end{tabular}

A relação das duas análises de sensibilidade e suas respostas nos gráficos expostos acima mostram semelhanças e discrepâncias entre as duas equações. Essas diferenças já eram esperadas, tendo em vista que cada equação apresenta particularidades quanto ao peso de seus fatores e configuração.

As principais semelhanças estão nos valores de coesão, ângulo de atrito interno do solo e espessura do solo. Essas três variáveis/parâmetros se mostram diretamente proporcionais ao FS. Da mesma maneira os fatores com valores indiretamente proporcionais coincidem nas duas equações e são 
representados pela declividade, nível freático, peso específico natural do solo e peso específico submerso/saturado do solo.

As semelhanças se limitam à proporcionalidade dos valores, as diferenças são muito mais significativas e podem ser percebidas tanto no comportamento individual das variáveis/parâmetros quanto no comportamento das equações. Individualmente os valores de coesão, nível freático, espessura do solo e ângulo de atrito têm respostas bem diferentes. Quando analisadas as respostas das equações, elas se diferem principalmente nas variáveis/parâmetros com maiores pesos no FS como o ângulo de atrito (segundo fator mais sensivel na equação 2) e o nível freático (segundo fator mais sensível na equação 1). Isso mostra um direcionamento tomado pelos autores das equações quanto aos fatores considerados mais importantes em suas análises, evidenciando a adaptação das equações às particularidades de cada região.

\section{Considerações Finais}

A análise desenvolvida nesse trabalho abarca apenas duas equações de FS, no entanto muitas outras foram desenvolvidas e são largamente aplicadas. O resultado exposto aqui mostra que deve existir um estudo prévio das equações de FS que serão utilizadas em estudos de estabilidade de encosta afim de se escolher aquela que se adequa melhor à área de aplicação. Dessa maneira as características naturais da área mais relevantes estarão em consonância com as variáveis/parâmetros de maior peso da equação escolhida, levando, consequantemente, à resultados mais próximos aos verificados na área de estudo.

\section{Referências}

AGAM, M. W.; HASHIM, M. H. M.; MURAD, M. I.; ZABIDI, H. Slope Sensitivity Analysis using Spencer's Method in Comparison with General Limit Equilibrium Method. Procedia Chemistry, n 19, p 651-658, 2016.

FIORI A.P. E CARMIGNANI L. Fundamentos de mecânica dos solos e das rochas: aplicações na estabilidade de taludes. $2^{\text {a }}$ ed., Curitiba, Editora UFPR, 604 p, 2009.

HAMOND, C.; HALL, D.; MILLER, S.; SWETIK, P. Level I Stability Analisys Documentation for Version 2.0. Departamento de agricultura dos Estados Unidos. Serviço Florestal. 1992.

LOPES, F. C. A. Avaliação da influência da distribuição espacial da espessura do solo e do nível freático na estabilidade de encosta. 2013. 123 f. Dissertação (Mestrado em Geografia) - Universidade Federal do Paraná, Curitiba, Paraná.

SIDLE, R. C. A dynamic model of slope stability in zero-order basins. Erosion and Sedimentation in the Pacific Rim (Anais do Corvallis Symposium) August, 1987. IAHS Publ . no. 165.

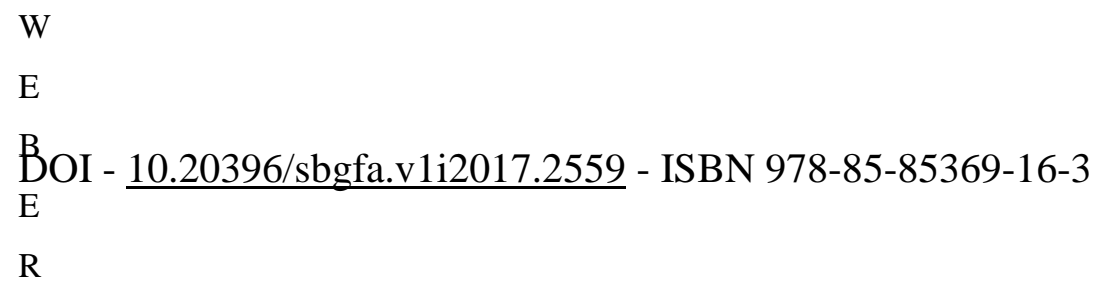

